\title{
Synchronization of Multiple Pulsed Alternators Discharging into an EM Launcher
}

\author{
Dr. Sid Pratap SM IEEE, Dr. Ray Zowarka, Mr. Tom Hotz, Mr. Scott Pish, Dr. Brian Murphy \\ The University of Texas at Austin Center for Electromechanics, Mail Code R7000, 1 University Station, Austin, Texas, 78712
}

\begin{abstract}
As the energy level in the projectile increases it becomes necessary to use multiple pulsed alternators discharging in parallel into the EM launcher. Another reason for having more than one machine is to compensate torque and gyroscopic effects of the pulsed alternator. This requires that machines be built in counterrotating pairs. These machines are identical in all respects except for their direction of rotation. A study was conducted to determine how the machines can be motored so that they stay in lock step in speed and phase as they are motored to full speed. The methods of connecting these multiple machines are discussed. The aim of the connection scheme is to allow the machines to naturally stay locked in speed and phase throughout its operating range. Sensitivity of the performance of these machines to small variations in the machine parameters, which is to be expected in the machines, is discussed. Sensitivity of the discharge performance to small phase angle mismatches due to tolerances is also discussed.

To verify the conclusions of the study an experiment was performed on two identical $50 \mathrm{kVA}$ machines discharging into a low impedance load. The motoring system that is discussed in the study was implemented in this experiment which kept the machines in lock step. This motoring system is described. Thereafter discharges were made at various speeds and field current levels. Phase angle mismatches were introduced between the two machines to see how it affected current sharing. The results and conclusions of these tests are presented in this paper.
\end{abstract}

\section{INTRODUCTION}

The practical implementation of pulsed, non-linear, shipboard loads require that the power supply (i.e. the compensated pulsed alternator) is torque compensated and imparts a minimum gyroscopic moment to the platform. Also, as the energy demanded by the pulsed loads increases, building multiple machines that operate in tandem are required to keep the size of the power supply manageable. To achieve this objective it is envisioned that an even number of machines be used that operate in unison and each pair has rotors spinning in an opposite sense. When we accept this basic tenet, we have to answer the question of synchronizing multiple machines. In a larger sense, synchronizing power generators with the grid is a routine operation conducted by the power industry [1], however timing and operational issues make this same synchronizing operation significantly more critical in an EM gun system. These issues have been raised in by Eccleshall and Pratap [2]. In this paper specific cases and their sensitivities have been studied and a motoring scheme is suggested that helps the process of synchronization. Shepard [3] and Chen [4], have done some work in this area however they used two machines that were very dissimilar: under discharge one acted as a motor and the other as a generator so current sharing between the generators was not established.

Fig. 1 shows two possible ways in which an EM Gun can be connected to the machines (through the rectifiers). Fig. 1a shows the two machines in parallel, they generate the same voltage i.e. the load voltage but share the load current. Fig. 1b shows the two machines in series, each generates half the load voltage but carries the full load current. The preferred approach is the one of Fig. 1a. This preference comes from some practical considerations. Fig. 1b configuration would require fewer turns in the armature winding so that the voltage generated is lower. The added cross section of the conductors would enable each of the machines in Fig. 1b to carry twice the current of the configuration in Fig. 1a. However the armature conductors in this case would have an aspect ratio that is not achievable in reality. The width of this conductor would be very large compared to its height and cannot be manufactured. Therefore for most of the studies here the parallel (share load current) option will be investigated.
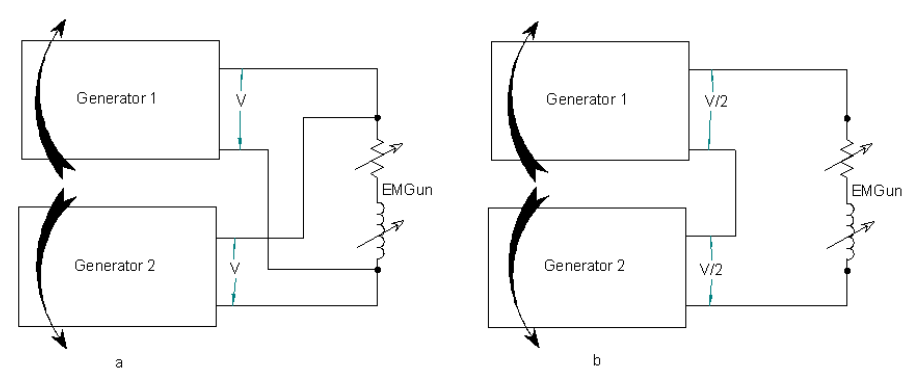

Fig. 1 (a) and (b). Two possible ways in which the dual machine system can be connected to the load

Fig. 1 specifies how the machines connect to the load, however it must be remembered that each of the two machines also charge their own field coils in a self-excitation mode. There are hence various ways that the field coils can be connected for charging. Fig. 2 shows two possible ways that the field coil can be connected. 


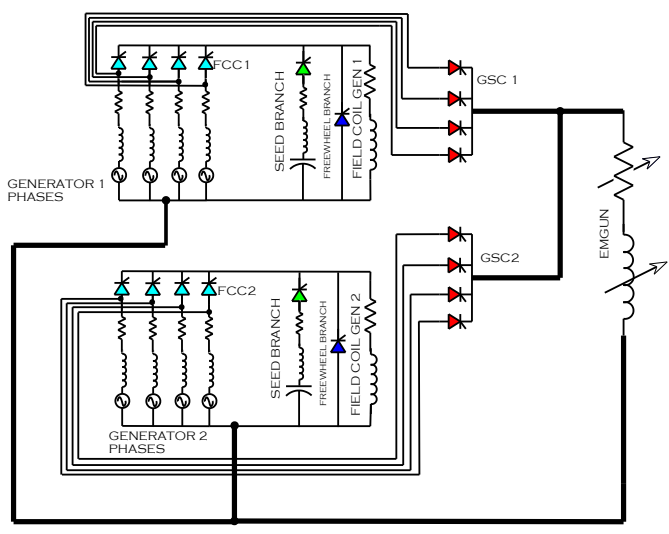

(a)

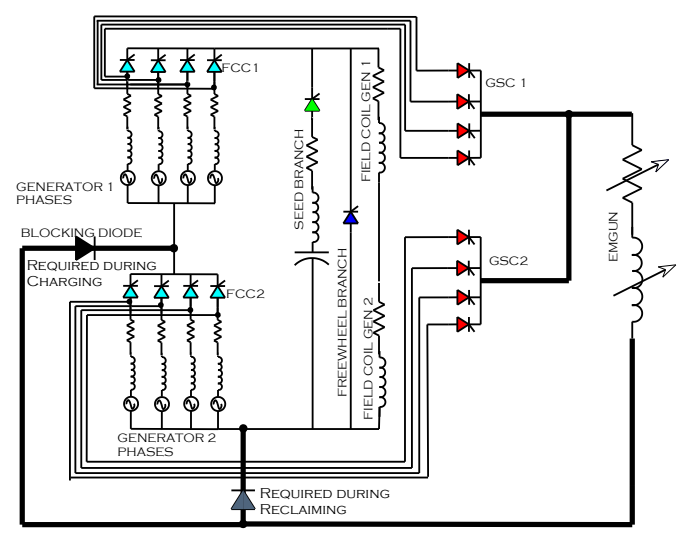

(b)

Fig. 2 (a) and (b). Two possible ways of connecting the field coils to the machines

Fig. 2a shows the field coils charging independently and Fig. $2 b$ shows the two field coils connected in series being charged by the two machines in series. The rate of charge in the two cases is identical. If there are some variations in the field coil parameters the scheme of Fig. 2a will result in correspondingly different charge rates and some loss of synchronism during the field charging process. Fig. $2 \mathrm{~b}$ shows a scheme that is immune to slight variations in the field coil parameters. However it is necessary to provide blocking diodes in the load discharge circuit (as shown) so as not to short the output of the field rectifier of the lower machine. These diodes must be capable of carrying half the gun current each and is nontrivial. The scheme of Fig. 2a is therefore preferred despite its drawbacks. A sensitivity study was conducted to see how much parametric variations can be tolerated by the systems.

Another scheme that must be investigated is the one shown in Fig. 3. Here the corresponding phases of the two machines are connected in parallel before connecting them to the rectifier. This allows circulating currents to flow freely between the two machines allowing them to align themselves should misalignment between the phases occur.

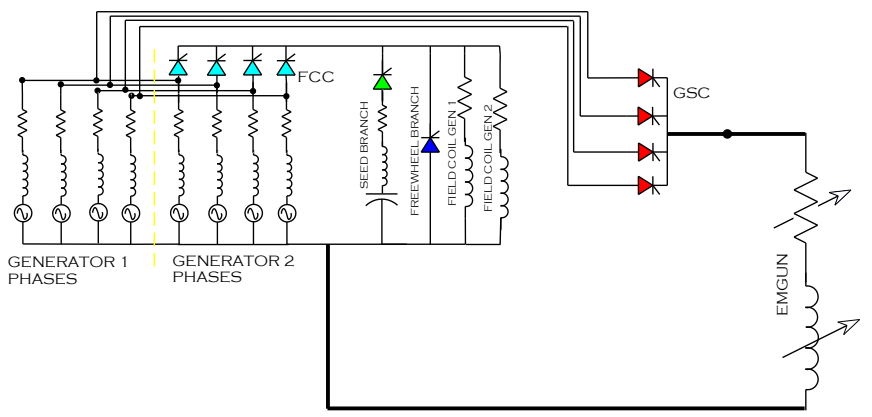

Fig. 3. Truly parallel scheme - machine phases are parallel before connecting to rectifier.
It must be noted that all the schemes being considered require that the machines be brought up to almost equal speed and phase before initiating the seed current into the field coils. Two schemes have, at this stage, made the initial screening process: Fig. 2a and Fig. 3. Both of these correspond to the basic parallel scheme of Fig. 1a.

The comparison of the performance of these two schemes was conducted under an earlier program. The comparison took into account effects such as slightly different inertias $(2 \%-3 \%)$, some differences in the field coil parameters, and some initial offsets in the rotor speed and phase. From this comparison of the two systems it was concluded that the truly parallel system is the most robust. The ability for synchronizing currents to freely flow between the machines allows them to stay in step under a wide variety of parametric variations. This system of paralleling was therefore selected as the baseline for further investigations. It must be reiterated that the motoring system must bring the two machines fairly close in speed and phase before the discharge can begin.

So far the majority of tests performed with pulsed alternators have only used a single machine. There is therefore no experience with operation of the pulsed machines in parallel. This task was undertaken in this program on a smaller scale using conventional $50 \mathrm{~kW}$ generators connected in a manner representing pulsed operation. The two machines were identical in all respects. The experimental setup of the generators and the synchronizing motoring system is shown in Fig. 4. The motors were each rated at $7.5 \mathrm{~kW}$ and interfaced with a vector control inverter. The two motors and inverters were arranged in a master-slave arrangement. There was a supervisory controller that ensured that the slave tracked the master at all times during motoring. 


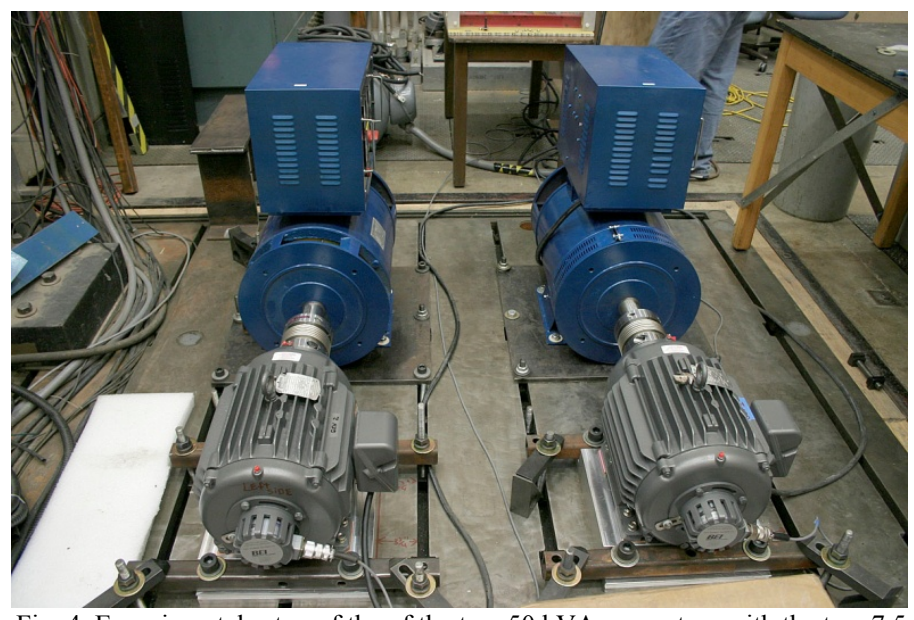

Fig. 4. Experimental setup of the of the two $50 \mathrm{kVA}$ generators with the two 7.5 $\mathrm{kW}$ synchronizing motors

\section{MOTORING SYSTEM}

\section{A. $\quad$ Motors and Controller}

As already mentioned, the motoring system is a very critical part of the dual machine system. The task of the motoring system is to not only bring the machines to the required speed but to do so ensuring that the two machines maintain the same speed and phase through the motoring. This induction motor was rated for $7.5 \mathrm{~kW}$ and was operated at $460 \mathrm{Vrms}$ line to line voltage. It was capable of operating at a maximum speed of 5000 $\mathrm{rpm}$. It had a moment of inertia of $0.09 \mathrm{~kg}-\mathrm{m}^{2}$. The motor and controller were purchased from Baldor. The selection of the power rating of the motor was based on dynamic considerations. One could motor the generator much slower with a lower power motor, however, a lower power motor would also have a smaller shaft. This would lower the torsional stiffness of the shaft system and since the natural frequency of the two inertia system is dependent on the torsional stiffness of the shaft this would cause undue oscillations that could potentially interfere with the control system and the synchronization process. This is an important consideration and will be revisited in the section on the coupling selection.

There were two controllers one for each motor. These two controllers performed the task of providing an input voltage profile through a pulsed width modulation (PWM) algorithm. The input to each of the two PWM controllers came from the motion controller which received shaft encoder inputs from the two motors. The speed as well as position was fed back to the torque calculator in the supervisory controller through a proportional integral (PI) loop. Excellent tracking was observed with this scheme.

\section{B. Coupling}

There were two sets of couplings ordered for this test. One variety was the Lovejoy flexible couplings and the other one was the $\mathrm{R}+\mathrm{W}$ Bellows coupling. The flexible coupling was not as stiff as one would need for this experiment but it was available on short order and served the purpose of checking out each generator independently. The other problem of the flexible coupling was that it was prone to a larger backlash on torque reversal and therefore could potentially interfere with the controller and make it difficult to lock phases.

The torsional stiffness of the Bellows BK2-800 coupling is given as $780 \mathrm{kN} / \mathrm{rad}$. The moment of inertia of the two rotors. motor and generator, are 0.09 and $2.1 \mathrm{~kg}-\mathrm{m}^{2}$ respectively. This gives a torsional natural frequency of the two inertias at about $480 \mathrm{~Hz}$ according to the formula $f=\sqrt{\frac{k\left(J_{m 1}+J_{m 2}\right)}{J_{m 1} J_{m 2}}}$. Here ' $\mathrm{k}$ ' is the torsional stiffness and $\mathrm{J}_{\mathrm{m} 1}$ and $\mathrm{J}_{\mathrm{m} 2}$ are the two moment of inertias. This torsional frequency is high enough so as not to interfere with the controller. Typically, this should be 5 to 10 times the loop bandwidth due to the rise time requirements.

\section{OTHER COMPONENTS OF THE EXPERIMENT}

\section{A. $\quad$ The Generators}

It was difficult to obtain two identical pulsed alternators for these tests due to time, cost, and other constraints. Our approach was therefore to use two conventional machines that operated in the pulsed mode. A variety of options were considered which were complicated by the fact that the two machines needed to have brushes and slip rings just as the pulsed alternators do. After careful evaluation of several options, a 50 $\mathrm{kW}$ generator was selected. These were manufactured in China by the QinFeng Electric Machinery Co., Ltd. The nameplate information on these machines is shown in Table 1. They were designed for an output voltage of $400 \mathrm{Vrms}$ line to line and were rated for a maximum speed of $1800 \mathrm{rpm}$. The output terminals included the neutral together with the three phases - an important aspect for the experiments.

This generator came from the manufacturer with a rectifier and an automatic voltage regulator for the field coil current and the machine was self excited. For the purpose of this experiment the self excitation was disabled and the machine was excited externally with a separate power supply through the brushes and slip rings.

Table 1. Nameplate rating of generators

\begin{tabular}{|l|c|}
\hline Parameter & Value \\
\hline Power Rating & $50 \mathrm{~kW}$ \\
\hline Voltage (line to line rms) & $400 \mathrm{~V}$ \\
\hline Full Load Current (rms) & $90.2 \mathrm{~A}$ \\
\hline Power Factor & 0.8 \\
\hline Frequency & $60 \mathrm{~Hz}$ \\
\hline Machine Speed & $1800 \mathrm{rpm}$ \\
\hline Insulation Class & 'B' \\
\hline
\end{tabular}




\section{$B$.}

\section{Switches}

An important aspect of the tests is to sequentially discharge each phase of the generators into the near short circuit load. Simulations of the $50 \mathrm{~kW}$ generator were used to estimate the current and voltage requirements on the switches. The switches, one for each phase pair, were selected based on the pulsed duty rating and the action limits. The switch selected was the Powerex T8K7453503DH. The three sets of switches for the three phases is shown in Fig. 5. Two inputs are seen for each phase, one from each generator. The phases are connected together before the switch, which allows circulating currents between the machines.

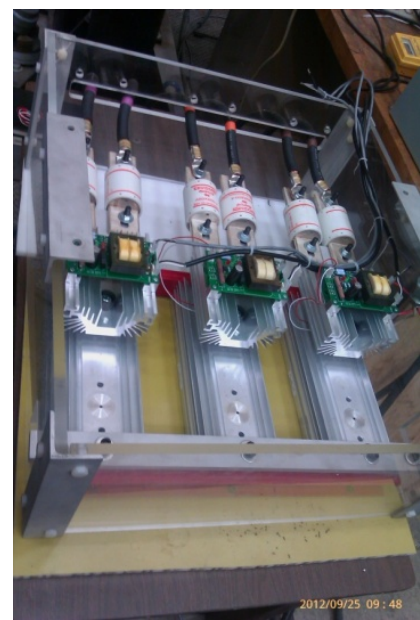

Fig. 5. Switch assembly with trigger and fuses

\section{EXPERIMENTAL SETUP}

Fig. 6 shows the circuit schematic for the test. This also shows the quantities that were measured. The field coil for the two generators were connected in series to ensure that the field current in the two machine were identical. The load in this case was simply a resistor $(0.25 \Omega)$ into which a set number of pulses were discharged.

The sequence of operation for motoring was as follows. One starts with the generators at zero speed. Thereafter the supervisory controller determines angular position of the master and slave rotors and moves the rotor position so that it is in lock step with the master rotor. Thereafter the motoring commences using vector control [5] on the induction motors, and at all times the slave remains in lock step with the master. Fig. 7 shows typical motoring speed curves for both machines. At around $30 \mathrm{~s}$ the drive goes into freewheel mode. In this case (Fig. 7) the discharge was not initiated nor was the field coil energized. As can be seen as soon as the freewheeling of the rotors commences the two rotors lose lock-step and deviate in speed.

Fig. 8 shows a speed curve for the two generators with the field coils initiated just prior to the discharge at $0 \mathrm{~s}$. Thereafter the rotors go into a freewheeling mode. Then the switches are triggered (at $0 \mathrm{~s}$ ) and a discharge is initiated. The sharp drop in the two speeds is associated with the discharge energy being extracted from the rotor. The discharge stops within a fraction of a second $(100 \mathrm{~ms})$. At this point the field coil is still on. Note that so long as the field coil is on, the two rotors stay locked in speed. Around $10 \mathrm{~s}$ the field coil current is switched off and the rotors slowly deviate in speed as the drag on the two rotors is slightly different due to friction and windage losses. While the field coil is on, the circulating currents between the two machines flow to keep the phases locked. This suggests an operating sequence where after the discharge is completed the motoring system needs to re-engage at or around zero current in the field coil. This ensures that the rotor remains locked throughout the multiple sequential discharges that may be called for in the operating cycle.

The discharge currents in the three phases and the load is shown in Fig. 9. The current is equally shared between the two machines. This is to be expected since the two machines are phase and speed locked and carry the same field current.

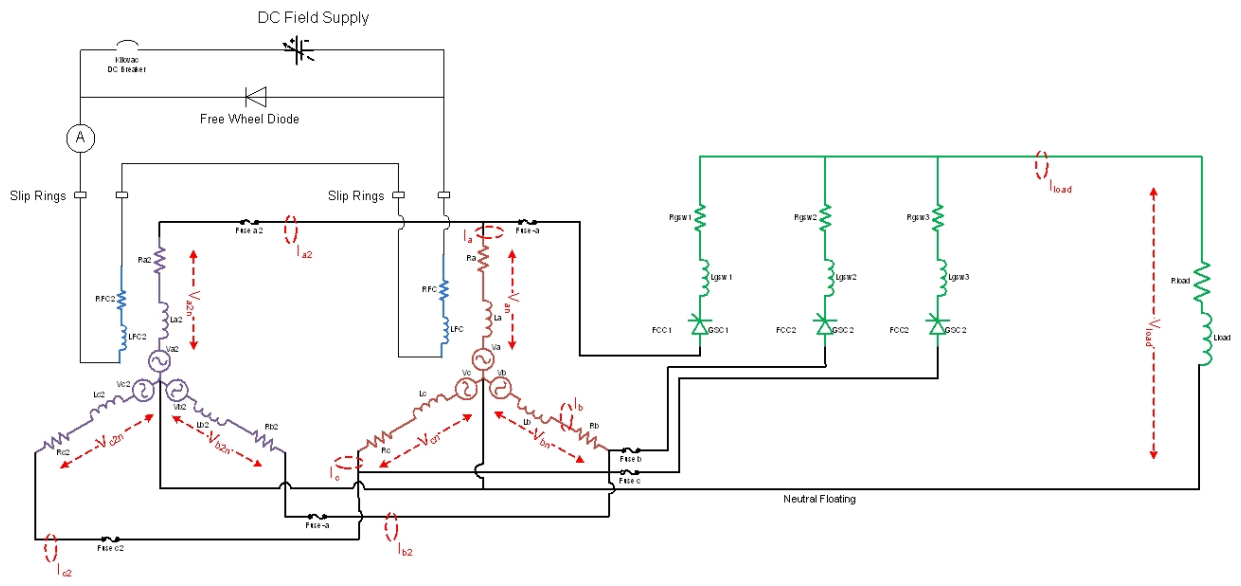

Fig. 6. Circuit schematic for the tests with the measurements that were recorded 


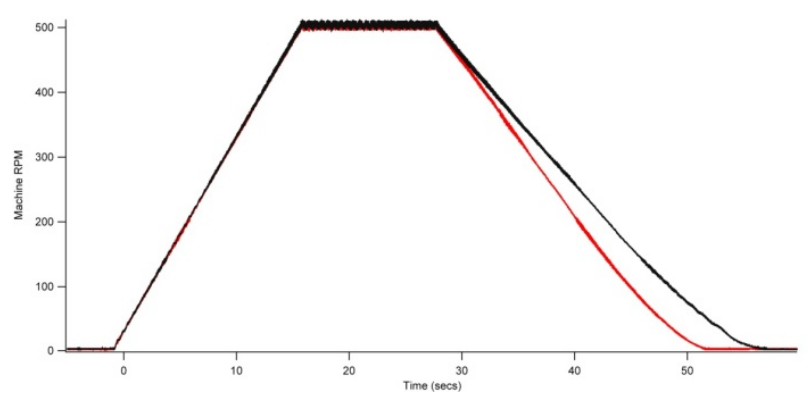

Fig. 7. Machine speeds of the two machines during spin up

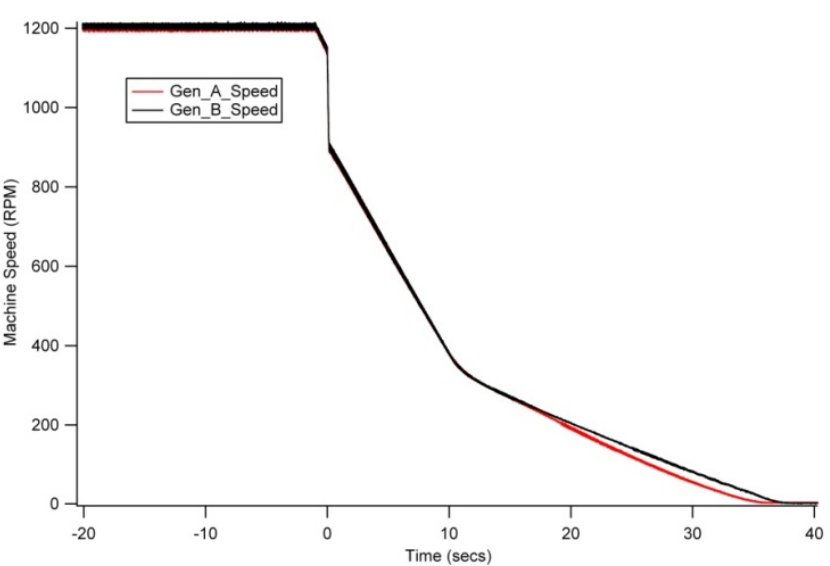

Fig. 8. The two generator speeds before, during and after discharge

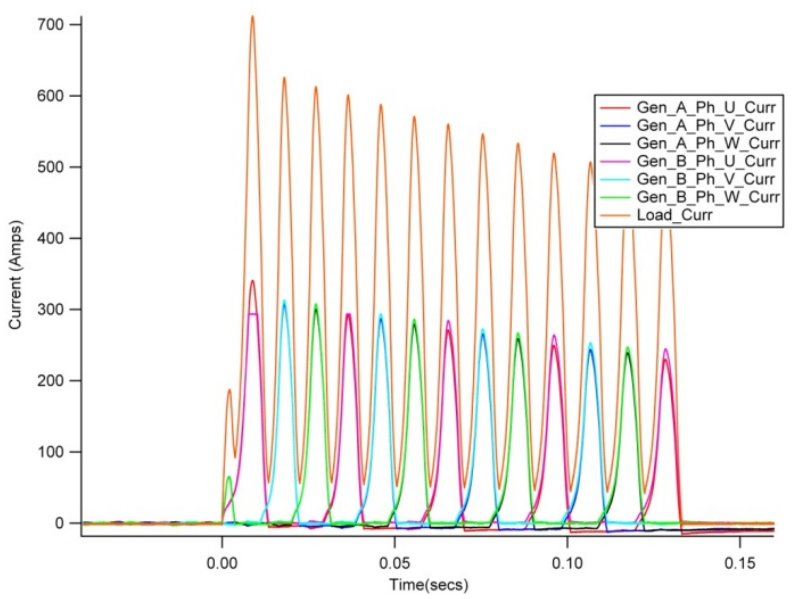

Fig. 9. Currents in each phase leg of both generators and the total load current

\section{A. Introduction of Phase Angle Errors}

To understand the sensitivity of the performance of the two generator system with phase angle errors these were introduced on purpose. These errors could enter the control system through the shaft encoder or even through the mounting of the shaft encoders and manufacturing tolerances. The slave rotor was asked to track the master rotor behind by an angle of $1^{\circ}$ electrical through $4^{\circ}$ electrical. Two set of tests were performed. In the first series of tests only the field coils were energized, but no discharge was initiated. In the second series of tests the discharge was initiated.

In the first series of tests the rotor speeds were observed and the circulating currents were recorded. The circulating currents were caused by the phase difference. These series of tests are summarized in Table 2. For the first test in this table, under freewheeling conditions the two rotor speeds were seen to oscillate around each other (Fig. 10) as the rotors to try to eliminate the phase difference between them with the circulating currents. Up to the $4^{\circ}$ electrical offset in the rotor angles the circulating currents were within manageable limits.

The second set of tests were performed with the angular offset and a discharge. The tests tried to gauge the amount of the current imbalance that would be introduced in the two machines when discharged in parallel. The results of these set of tests are shown in Table 3.

\section{CONCLUSIONS}

The experiment demonstrated that two machines can be locked in speed and phase using off the shelf drive technology. The pulsed discharge of the two machine system was successful with the load current being shared equally between the two machines for the range of phase displacements studied.

This experimental set up serves as a test bed to check out the performance of other topologies such as paralleling the machines on the dc side rather than the ac side and using other control strategies and for synchronizing the two machines. One could also use this test bed to perform other tests such as self excitation and discharge in parallel. Considering that the machines are relatively inexpensive a set of 4 machines can be used to test the system with more than multiple pairs of machines.

Having done this benchmark test on conventional machines helps to set the stage to observe the behavior of two air pulsed alternators as they are discharged in parallel. 
Table 2. Tests without discharges to observe the circulating currents with the phase displacements

\begin{tabular}{|c|c|c|c|l|}
\hline $\begin{array}{c}\text { Test } \\
\#\end{array}$ & $\begin{array}{c}\text { Machine } \\
\text { Speed } \\
\text { (rpm) }\end{array}$ & $\begin{array}{c}\text { Field Current } \\
\text { (A) }\end{array}$ & $\begin{array}{c}\text { Phase } \\
\text { Displacement } \\
\text { (electrical degrees) }\end{array}$ & \multicolumn{1}{|c|}{ Comments } \\
\hline 1 & 500 & 1.0 & $1^{\circ}$ & $\begin{array}{l}\text { Peak circulating currents up to 2 amps, machine speeds oscillate when they } \\
\text { freewheel as the machines ring around each other as they attempt to correct } \\
\text { the phase difference }\end{array}$ \\
\hline 2 & 1200 & 1.3 & $1^{\circ}$ & Peak circulating currents up to 3 amps. \\
\hline 3 & 1200 & 1.3 & $2^{\circ}$ & Peak circulating currents up to 4 amps. \\
\hline 4 & 1200 & 1.3 & $3^{\circ}$ & Peak circulating currents up to 5.3 amps. \\
\hline 5 & 1200 & 1.3 & $4^{\circ}$ & Peak circulating currents up to 6 amps. \\
\hline 6 & 1200 & 0.5 & $2^{\circ}$ & Peak circulating currents up to 4 amps. \\
\hline
\end{tabular}

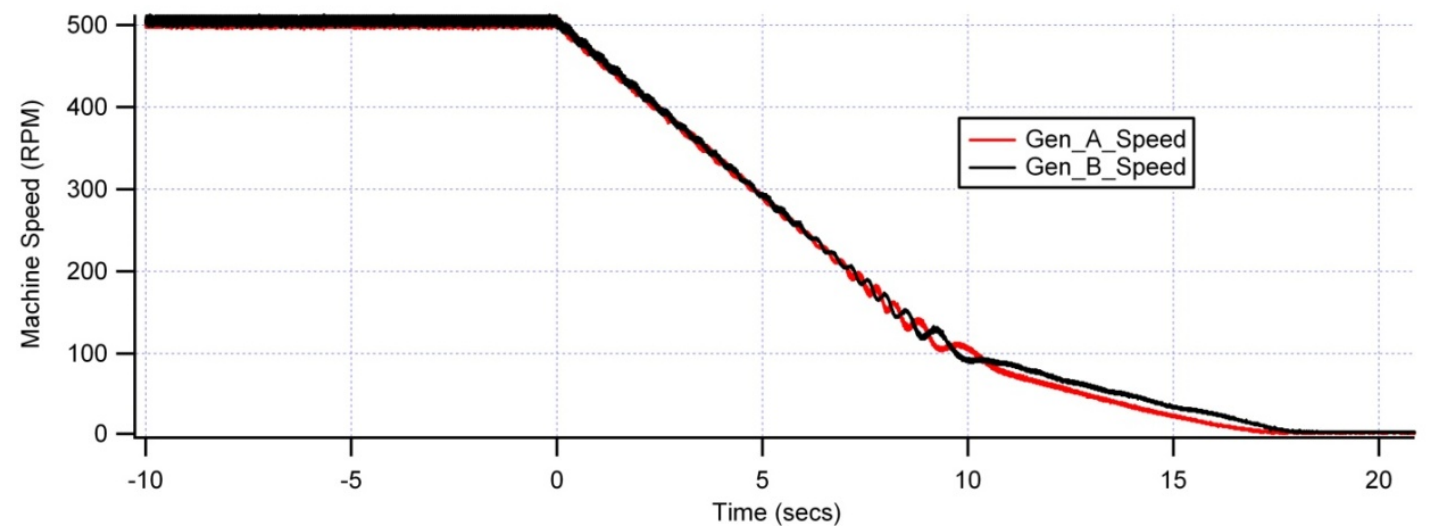

Fig. 10. Machine speed - clearly showing the machine speeds oscillating to eliminate the phase displacement

Table 3. Tests with discharges and phase displacements

\begin{tabular}{|c|c|c|c|l|}
\hline $\begin{array}{c}\text { Test } \\
\#\end{array}$ & $\begin{array}{c}\text { Machine } \\
\text { Speed } \\
(\mathrm{rpm})\end{array}$ & $\begin{array}{c}\text { Field } \\
\text { Current } \\
(\mathrm{A})\end{array}$ & $\begin{array}{c}\text { Phase } \\
\text { Displacement } \\
\text { (electrical degrees) }\end{array}$ & Comments \\
\hline 1 & 1200 & 1.0 & $1^{\circ}$ & Current sharing between machines was good \\
\hline 3 & 1200 & 1.0 & $2^{\circ}$ & Current sharing between machines was good \\
\hline 4 & 1200 & 1.0 & $3^{\circ}$ & Current sharing between machines was good \\
\hline 5 & 1200 & 1.0 & $4^{\circ}$ & Current sharing between machines was good \\
\hline
\end{tabular}

\section{ACKNOWLEDGMENTS}

This work was performed with funding from the Office of Naval Research under contract no. FSU\# 225000-520-029363/ ONR N00014-10-1-0973.

\section{REFERENCES}

[1] Alternating Current Machines $3^{\text {rd }}$ Edition, by A. F. Puchstein, T. C. Lloyd and A. G. Conrad, 1964, John Wiley \& Sons. Pgs 527-534.

[2] D. Eccleshall and S. Pratap, "Comparison of a single pulsed alternator with two or more in parallel for driving a railgun," IEEE Transactions on Magnetics, Vol 37, No. 1, January 2001, pgs 473-475.

[3] N. Shepard, Y. Y. Chen, and J. Mallick, "Physical Scale Model Alternators as a Pulsed-Power Source Simulator." IEEE Transactions on Magnetics, Vol. 43, No. 1, Jan. 2007, pgs 265-269

[4] Y. Chen, N. Shepard, J. Mallick, "Experimental Results from a Physical Scale Model Alternator Pair as a Pulsed Power," IEEE Transactions on Magnetics, Vol. 43, No. 1, Jan. 2007, pgs 230-233.

[5] Vector Control and Dynamics of AC Drives (Monographs in Electrical and Electronic Engineering, No 41) by D. W. Novotny and T. A. Lipo., Oxford University Press 